\title{
Targeting of p53-Transcriptional Dysfunction by Conditionally Replicating Adenovirus Is Not Limited by p53-Homologues
}

\author{
Florian Kühnel' ${ }^{1}$ Engin Gürlevik1, Thomas C Wirth², Nina Strüver1, Nisar P Malek', \\ Martina Müller-Schilling ${ }^{3}$, Michael P Manns ${ }^{1}$, Amancio Carnero ${ }^{4}$, Lars Zender ${ }^{1}$ and Stefan Kubicka ${ }^{1}$

\begin{abstract}
${ }^{1}$ Department of Gastroenterology, Hepatology and Endocrinology, Medical School Hannover, Hannover, Germany; ${ }^{2}$ Department of Microbiology, University of lowa, lowa City, lowa, USA; ${ }^{3}$ Department of Gastroenterology, Ruprecht-Karls-University of Heidelberg, Heidelberg, Germany; ${ }^{4}$ Experimental Therapies Programme, Centro National de Investigaciones Oncologicas (CNIO), Madrid, Spain
\end{abstract}

A hallmark of human tumors is the loss of p53 or its transcriptional functions. In this study, we describe the generation of the conditionally replicating adenovirus Adp53sensor for the treatment of p53-dysfunctional tumors. p53-selective attenuation of viral replication was achieved by using p53-dependent expression of the transcriptional repressor Gal4-KRAB that was directed against the adenoviral E1A locus. Adp53sensor shows efficient replication in p53-dysfunctional, but not in p53active cells. In p53-dysfunctional cells, p53-analogous transcriptional activity by other p53 family members was not sufficient to compromise replication of Adp53sensor. In comparison with a genetically similar, but p53insensitive virus, Adp53sensor replication was inhibited after systemic infection of p53-wt-mice, but not in p53ko-mice thus confirming the correct function of the chosen approach. Adp53sensor showed efficient lytic and replicative properties in all investigated cells with p53dysfunction and successfully inhibited the growth of subcutaneous xenotransplants in vivo. We further demonstrated that intravenous injection of Adp53sensor lead to significantly reduced liver damage compared to the control virus. Together, our data show that Adp53sensor is an oncolytic, p53-selective adenovirus for efficient treatment of p53-dysfunctional tumors with a favorable toxicity profile. Moreover, Adp53sensor provides a strategy that should be applicable to other transcriptionally regulated DNA viruses.

Received 29 April 2009; accepted 5 December 2009;

published online 29 December 2009. doi:10.1038/mt.2009.298

\section{INTRODUCTION}

Tumor selectively replicating oncolytic viruses are a novel class of antineoplastic agents that hold promise to overcome limitations of conventional cancer therapy. For the generation of oncolytic viruses, tumor-specific promoters have been used to control E1A expression and viral replication. However, this approach is often limited by weak promoter activity and heterogeneous activity in different tumor subzones. Additionally, E1A accumulation due to basal promoter activity in normal cells can finally lead to replication onset and loss of selectivity, in particular at high multiplicities of infection. ${ }^{1,2}$ Consequently, alternative strategies are required that ensure stringent regulation of viral replication in response to crucial oncogenic pathway aberrations.

The tumor suppressor p53, which normally controls genomic stability and cell cycle control, is genetically or epigenetically altered in the majority of human tumors. p53-activity is induced following diverse genotoxic stimuli and results in transcriptional activation of proapoptotic genes, cell cycle arrest, and apoptosis., Because alterations of the p53-pathway leading to a loss of p53transcriptional functions are fundamental events in carcinogenesis, p53-transcriptional deficiency represents an ideal target for tumor therapy.

Therefore, p53-selective virotherapy has been addressed first by the use of the oncolytic adenovirus dl1520 (Onyx-015), an E1B-55k mutant originally designed to replicate specifically in p53-dysfunctional cells. However, E1B-55k is not only an important inhibitor of $\mathrm{p} 53$ but also exerts further important and versatile functions that support the viral replication cycle. ${ }^{5-7}$ Furthermore, the adenoviral E4orf1 has been shown to limit the oncolytic potential of E1B-55k-deleted oncolytic adenoviruses. ${ }^{8}$ Consequently, the replication of Onyx-015 is often severely compromised in p53-dysfunctional cancer cells and first experiences of clinical applications did not meet high expectations. To circumvent manipulations of key viral proteins such as E1B-55k, and to maintain the natural replication cycle of the virus, we constructed a p53-dependent oncolytic adenovirus without any alterations of early viral gene products. Tumor selectivity of this virus, designated Adp53sensor, is achieved by chemotherapy-triggered, p53dependent expression of the transcriptional repressor Gal4-KRAB, which in turn inhibits the expression of the early viral E1A gene. Our results show that Adp53sensor replication and oncolysis are restricted to p53-dysfunctional cells and is not compromised by endogenous activity of other members of the p 53 family that can potentially compensate the loss of p53-transcriptional functions. 
Adp53sensor showed improved replication kinetics and oncolysis in tumor cells, and enhanced tumor selectivity in vitro and in vivo compared to Onyx-015. Furthermore, Adp53sensor exhibited significantly increased therapeutic efficacy. In combination with chemotherapy, Adp53sensor efficiently inhibited growth of aggressive human tumor xenografts in vivo.

\section{RESULTS}

\section{Cell stress mediated by chemotherapy and/} or adenoviral infection induce expression of p53: a stress-induced pathway that facilitates chemotherapy-assisted, p53-dependent virotherapy

High adenovirus load or doxorubicin chemotherapy can trigger DNA damage pathways and subsequent activation of p53 in vivo. ${ }^{9,10}$ To investigate the intracellular $\mathrm{p} 53$-response after viral transduction, we treated p53-wt cells with a replication-incompetent adenovirus and/or doxorubicin. Both treatments were applied at low dose to reflect a physiologic environment for a p53-selective oncolytic adenovirus. Application of single agents led to upregulation of p53 in a dose-dependent manner and combined treatment further increased p53 levels (Figure 1a,b). We could recently demonstrate that chemotherapy does not compromise adenoviral replication and leads to improved oncolysis. ${ }^{11}$ Therefore, our results suggest that chemotherapy-assisted adenoviral infection sufficiently triggers an intracellular p53-response that could be exploited to regulate conditional adenoviral replication in a p53dependent manner. Consequently, we developed a vector concept (termed Adp53sensor, Figure 1c,d) harboring a p53-sensitive promoter for p53-restricted expression of the transcriptional repressor Gal4-KRAB. The repressor in turn binds to the cytomegalovirus (CMV)-promoter variant CMVgal and silences transcription of E1A. CMVgal consists of a 474 base-pair long CMV-promoter sequence equipped with internal and flanking clusters of Gal4binding sites to attract Gal4-KRAB as described previously. ${ }^{12}$ This genetic setup allows for inhibition of E1A expression under nonpermissive conditions and for high E1A levels under permissive conditions (Figure 1e). The CMVgal-promoter shows a higher transcriptional activity compared to the wild-type E1A-promoter, but is susceptible for efficient transcriptional repression by Gal4KRAB (Figure 1f).
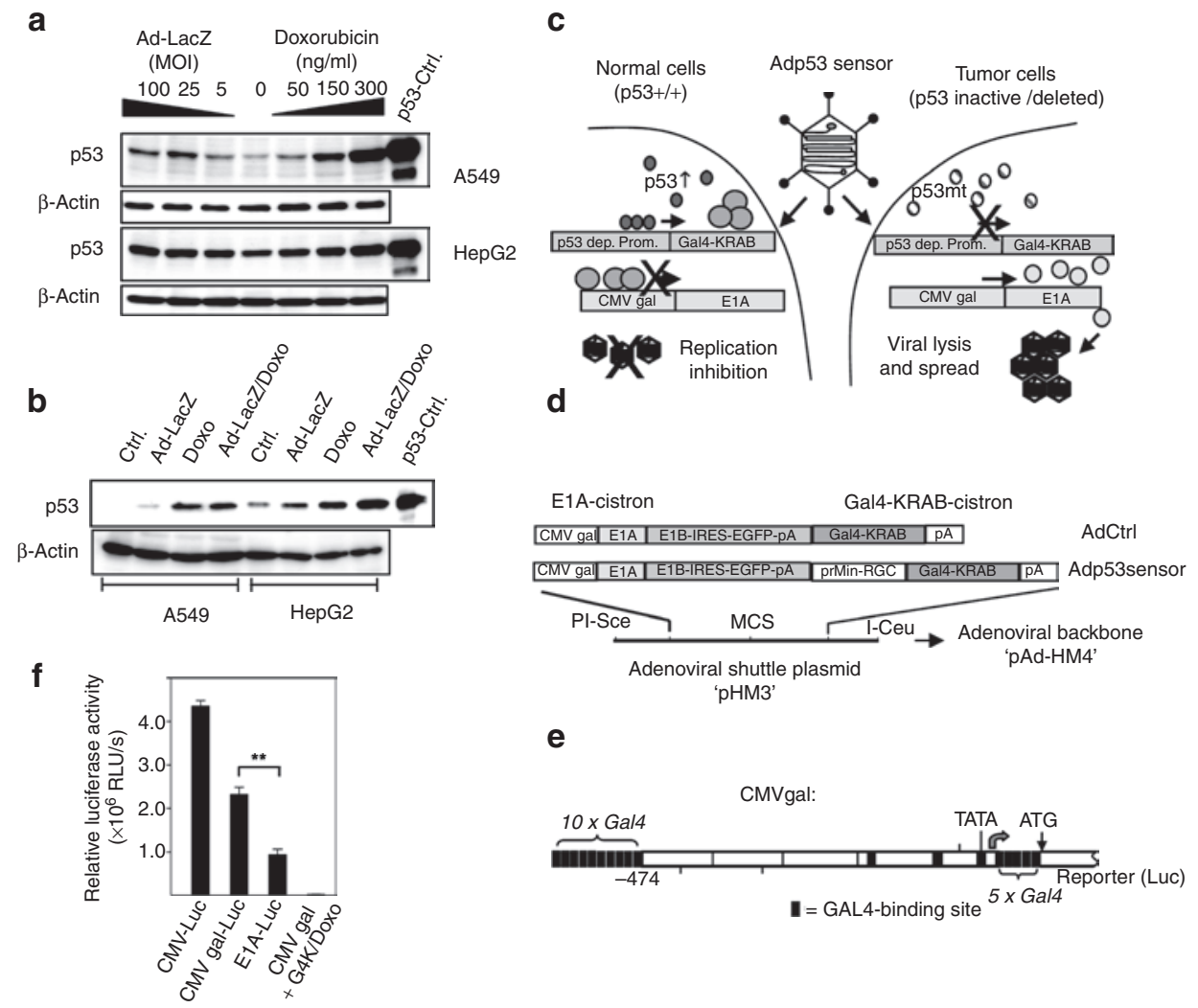

e

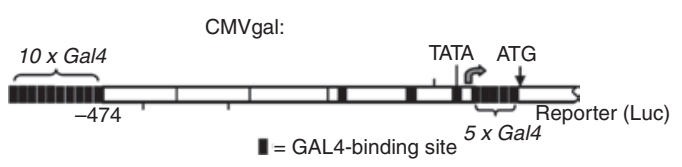

Figure 1 p53 activation upon adenoviral cell entry and chemotherapy provides the basis for the construction of Adp53sensor. (a) HepG2 and A549 were treated with indicated MOls/doses of Ad-LacZ or doxorubicin, or (b) with combinations of both agents (MOI $50 ; 200 \mathrm{ng} / \mathrm{ml})$. Western blot analyses revealed that p53 was induced by adenoviral infection and/or doxorubicin in a dose-dependent manner. (c) Strategy for selective adenoviral replication in p53-transcriptional-deficient cells. The bicistronic adenovirus Adp53sensor contains a p53-dependent promoter for control of the Gal4-KRAB repressor, functioning here as sensor element for the p53-transcriptional activity of the target cell. The second cistron contains the E1A gene under control of a GAL4-binding CMV-promoter mutant that can be silenced in the presence of Gal4-KRAB. (d) Illustration showing the genetic construction included in Adp53sensor and the p53-insensitive counterpart AdCtrl. (e) Illustration showing the genetic modifications included in the CMVgal-promoter to achieve efficient binding of Gal4-KRAB. (f) One microgram of the plasmids CMVwt-Luc, CMVgal-Luc, or prE1A-Luc were transfected into HepG2 cells to determine promoter activity by luciferase assays. Half a microgram of prMinRGC-GAL4KRAB was co-transfected for p53dependent expression of the repressor, and doxorubicin was administered at a final concentration of $200 \mathrm{ng} / \mathrm{ml}$. The data show that the promoter mutant CMVgal can be efficiently repressed by chemotherapy-triggered, p53-dependent expression of Gal4-KRAB. Ctrl, control; Doxo, doxorubicin; MOI, multiplicity of infection; RLU, relative light units; Luc, luciferase. ${ }^{* *} P<0.005$. 
a

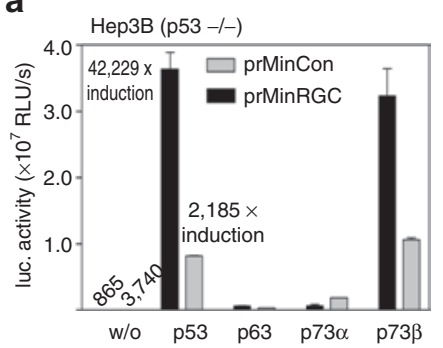

b

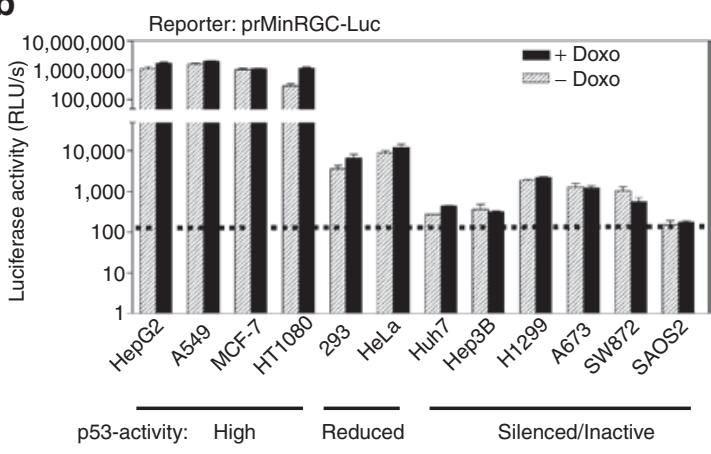

C

\begin{tabular}{|l|l|l|l|}
\hline & p53-status & p73-status & Comment \\
\hline HepG2 & wt & wt & \\
\hline A549 & wt & wt & \\
\hline MCF-7 & wt & wt & \\
\hline HT1080 & wt & unkn. & \\
\hline 293 & wt & unkn. & Ad5-E1 genes \\
\hline HeLa & wt & wt & HPV E6/E7 \\
\hline Huh7 & 220C & wt & \\
\hline Hep3B & del & wt & HBx \\
\hline H1299 & del & wt & \\
\hline A673 & wt & wt & Mdm2-overexpr. \\
\hline SW872 & wt/251C & unkn. & \\
\hline SAOS2 & del & wt & \\
\hline
\end{tabular}

d

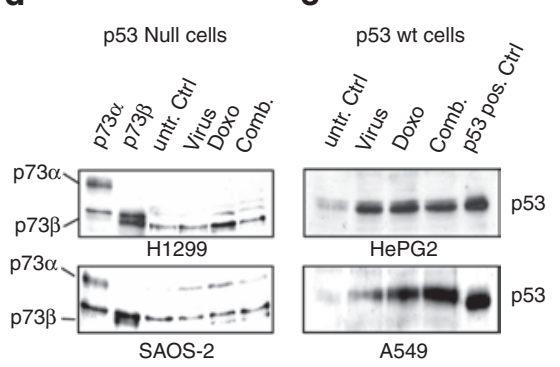

Figure 2 The prMinRGC promoter responds to p53 with a high signal/noise ratio and is not activated by endogenous levels of other p53 family members in tumor cells after adenoviral infection or chemotherapy. (a) Hep3B cells were co-transfected with $1 \mu \mathrm{g}$ prMinRGC-Luc or prMinCon-Luc, $0.3 \mu \mathrm{g} \mathrm{CMV-LacZ,} \mathrm{and} 0.05 \mu \mathrm{g}$ expression plasmid for $\mathrm{p} 53$ family members (pC53SN3, TAp63 $\alpha$, pcDNA3-HA-p73 $\alpha$, or pcDNA3-HAp73 $\beta$ ). Thirty-six hours after transfection, luciferase activity was determined. (b) Cells were transfected with $1 \mu \mathrm{g}$ prMinRGC-Luc and $0.3 \mu \mathrm{g}$ CMV- $\beta$ gal to investigate reporter activation by endogenous p53 or p53-homologues. Doxorubicin was administered at a final concentration of $200 \mathrm{ng} / \mathrm{ml}$. The reporter-free background level is illustrated by the dotted line. (c) Enumerates the known genetic p53/p73 status of the used cell lines. (d) SAOS-2 and $\mathrm{H} 1299$ cells were stimulated with doxorubicin $(200 \mathrm{ng} / \mathrm{ml})$ and/or Ad-LacZ at multiplicity of infection of 25 . Nuclear extracts were analyzed for p73 $\alpha$ and p73ß. (e) Nuclear extracts of p53-positive A549 and HepG2 after similar treatment were analyzed for p53.

\section{The p53-dependent promoter prMinRGC is not transactivated by other members of the p53 family upon chemotherapy and adenoviral infection in cancer cells}

To find a suitable p53-responsive promoter for application in Adp53sensor, we evaluated two artificial promoters, prMinCon and prMinRGC, that contain a CMV-minimal-promoter and either clusters of consensus or ribosomal gene cluster (RGC) sites for binding of $\mathrm{p} 53$. Both promoters can be strongly transactivated by overexpressed p53 (Figure 2a). However, the prMinRGC-promoter provided a favorable activation/background ratio and was therefore selected for inclusion in Adp53sensor. In contrast to $\mathrm{p} 53$, other members of the p53 family are rarely altered in cancer, but several isoforms of $\mathrm{p} 63$ and p73 (collectively termed TAp63 or TAp73, respectively) can recognize p53-DNAbinding sites and transactivate p53 target genes. ${ }^{13,14}$ Because it has been reported that TAp73 or TAp63 can be upregulated by chemotherapy or adenoviral infection, ${ }^{15-19}$ we investigated transactivation of $\mathrm{p} 53$-responsive promoters by overexpressed TAp63, TAp73 $\alpha$, and TAp73 $\beta$ (Figure 2a). Transactivation by TAp73 $\beta$ was comparable to p53, but TAp63 and TAp73 $\beta$ led only to low promoter activity. Because this responsiveness to TAp $73 \beta$ could potentially compromise a broad applicability of Adp53sensor, we investigated for prMinRGC-promoter activation in the context of endogenous levels of p53- and p53homologues in a panel of cell lines (Figure $2 \mathbf{b}$ ). The promoter was strongly activated in p53-wt cells, in particular in HepG2 and A549. In p53-wt cells with epigenetic inhibition of p53 (such as 293 or HeLa), reporter expression was significantly reduced or almost completely abolished (A673). We could not find any cell line with genetic p53-dysfunction that showed a significant reporter activation including those with intact p73 (Figure 2c). This observation was unexpected because at least H1299 and SAOS-2 cells have been reported to upregulate p73 upon genotoxic stress. ${ }^{20,21}$ Analysis of nuclear TAp73 levels in these cells after stimulation confirmed the accumulation of TAp73 $\beta$ and/or TAp73a (Figure 2d), though the observed protein levels seemed to be rather low. Consistently, it has been reported that $\mathrm{p} 73$ isoforms are expressed at lower level than uninduced p53, even after chemotherapeutic stimulation. ${ }^{22}$ In contrast, p53 strongly accumulated in the nuclei of stimulated p53-wt cells (Figure 2e). Not only low levels of endogenous TAp73 can explain the missing prMinRGC-activation in p53-dysfunctional cells, but also a neutralizing balance of p73 isoforms could be responsible for low promoter activation. The p73-gene also encodes isoforms $(\Delta \mathrm{Np} 73)$ that lack a transactivation domain and can negatively interfere with p53- and TAp73-dependent transcription. Therefore, we tried to shift the balance between endogenous p73 isoforms by an siRNA approach and investigated prMinRGCpromoter activation. For knockdown of all isoforms, siRNA was directed against the DNA-binding domain (DBD) of p73. siRNA against the transcription activation domain was applied to selectively interfere with transcriptionally activating isoforms (Figure 3a). In p53-wt cells, a p73-knockdown did not 


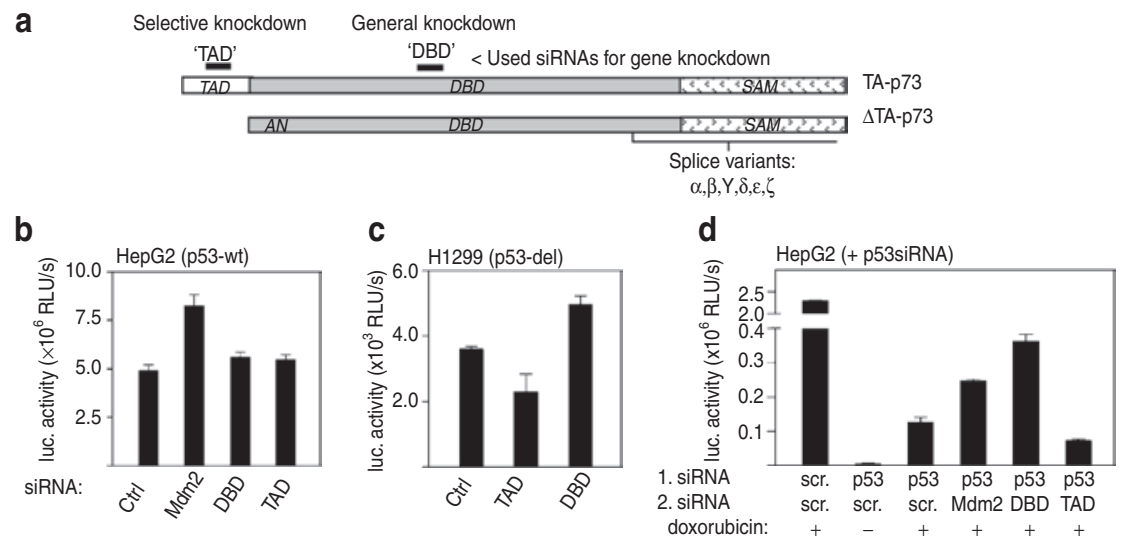

Figure 3 Endogenous p73 is unable to induce prMinRGC promoter activity in p53-negative cells, but is able to interfere with p53-dependent transcription under conditions of low p53-activity. (a) Scheme illustrating p73 isoforms and target sites of applied siRNAs for selective knockdown of transactivating isoforms (siRNA: "TAD") or general p73-knockdown (siRNA: "DBD"). (b) HepG2 cells (normal p53) were co-transfected with $1 \mu \mathrm{g}$ prMinRGC-Luc, $0.3 \mu \mathrm{g} \mathrm{CMV-LacZ,} \mathrm{and} \mathrm{siRNAs} \mathrm{as} \mathrm{indicated.} \mathrm{Doxorubicin} \mathrm{was} \mathrm{applied} \mathrm{at} \mathrm{a} \mathrm{concentration} \mathrm{of} 200 \mathrm{ng} / \mathrm{ml}$. After $48 \mathrm{hours,} \mathrm{cell} \mathrm{luciferase}$ assays were performed and $\beta$-gal-normalized. (c) p53-deleted H1 299 cells were treated and analyzed likewise. (d) HepG2 cells were additionally cotransfected with siRNA against p53. Analyses were performed as described before.

interfere with p53-dependent induction of prMinRGC-activity suggesting that fully activated p53 dominates the transcriptional activation (Figure 3b). In p53-negative H1299 cells, we observed a reduced reporter activity after selective knockdown of activating isoforms, but increased activity after silencing all p73 isoforms suggesting that the simultaneous presence of TAp73 and $\Delta \mathrm{Np} 73$ isoforms results in a net inhibition of the promoter (Figure 3c). We then investigated whether a p73-knockdown is able to modulate p53-dependent transcription under conditions of attenuated p53-activity in p53-wt cells (Figure 3d). siRNAmediated reduction of the p53-response by p53-directed siRNA and simultaneous silencing of all p73 isoforms reconstituted prMinRGC-activation to $20 \%$ of the full activity, a level that could not be reached by silencing of $\mathrm{Mdm} 2$, the physiological inhibitor of p53. These data suggest that endogenous p73 is able to efficiently interfere with attenuated p53-dependent transcription for the purpose of fine-tuning the transcriptional response. However, the data show that p73 is neither able to interfere with a fully developed p53-transcriptional response nor capable to significantly induce prMinRGC promoter activity under conditions of p53-dysfunction.

\section{Adp53sensor replicates in a p53-dependent, but p73-independent manner}

To investigate p53-dependent regulation of adenoviral replication, we used Adp53sensor in comparison to AdCtrl, a genetically similar, but p53-nonresponding control virus that lacks the prMinRGC-promoter. Consequently, the contribution of p53 or p53-analogous activity to conditioned replication and lysis can be directly determined by comparison of these two viruses in any given cell line. At first, we investigated E1A expression after viral infection of cell lines with different p53 status (Figure 4a). Consistent with our hypothesis, p53-dysfunctional cells expressed similar levels of E1A after infection with Adp53sensor or AdCtrl, and Gal4-KRAB expression could not be observed. In contrast, Gal4-KRAB was expressed after infection of p53-wt cells with Adp53sensor, that was further triggered by increasing doses of doxorubicin. The inverse correlation between E1A and Gal4KRAB levels not only indicates the correct function of the p53dependent control loop in Adp53sensor, but also underlines the usefulness of chemotherapy for additional triggering.

To investigate whether Adp53sensor replication and cytolysis is selectively inhibited in the presence of active p53, we examined the morphology of cells with different p53 status following infection (Figure 4b). Doxorubicin-pretreated HepG2 cells retained intact morphology 5 days after Adp53sensor infection, whereas AdCtrl-infected cells showed a strong cytopathic effect. Because both vectors effectively lysed p53-mutant cells, these observations suggest that Adp53sensor indeed functions as a p53-dependent oncolytic virus. Titration of the viral progeny (Figure 4c) revealed significantly reduced Adp53sensor titers in HepG2 cells compared to AdCtrl, showing that differential cytolysis correlated with the inhibition of viral replication. These data suggest that p53-dependent silencing of E1A expression inhibits adenoviral replication and lysis of infected cells. Investigation of viral replication in additional cell lines confirmed that Adp53sensor was selectively attenuated in p53active cells and that attenuation was significantly increased by doxorubicin (Figure 4c). Adp53sensor produced significantly less viral particles in p53-normal cells than Onyx-015. This finding was independent of chemotherapy application. Because Adp53sensor and AdCtrl replicated equivalently in all p53-dysfunctional cells, we could not find any correlation to the cellular p73-status. In general, Adp53sensor showed significantly improved replication compared to Onyx-015 in most p53-dysfunctional cells. Equivalent replication of both vectors could only be observed in Hep3B cells. This can be explained by expression of the $\mathrm{HBV}$-derived transactivator protein $\mathrm{HBx}$ in Hep3B cells that has been reported to support adenoviral replication. ${ }^{23}$ Taken together, our results demonstrate that Adp53sensor is selectively attenuated in response to activated p53 but is able to efficiently replicate in p53-dysfunctional cells in vitro. To further confirm these findings in vivo, conditional replication of Adp53sensor was investigated in a subcutaneously grown, 


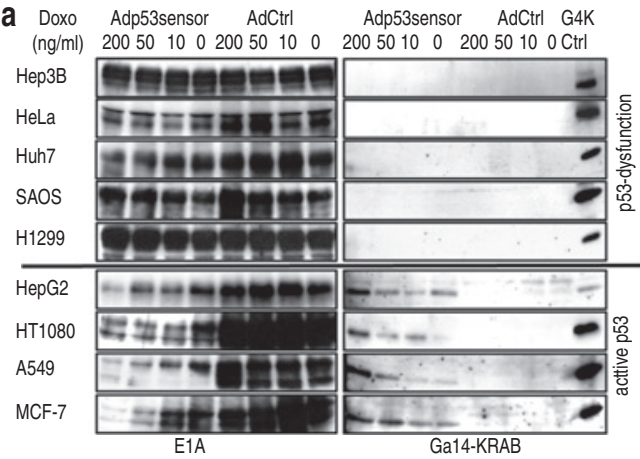

b

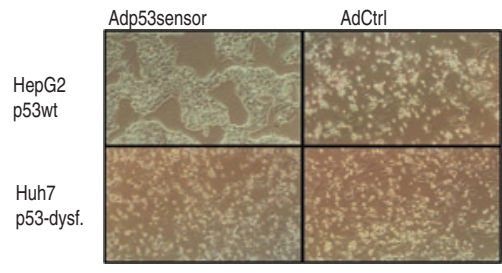

d

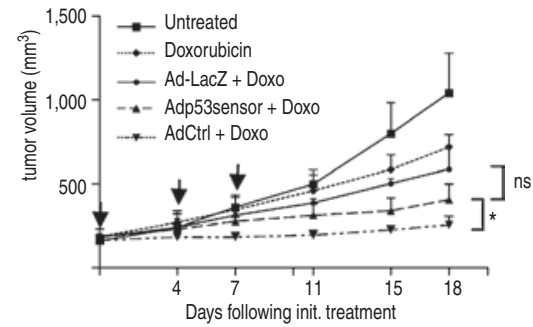

C

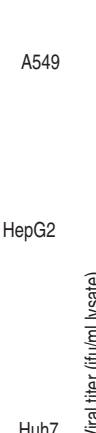

Huh7
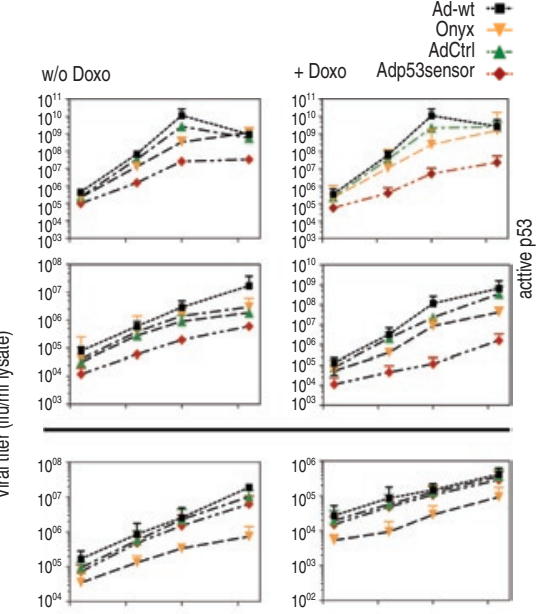

H1299

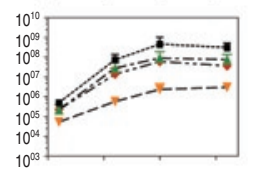

SAOS

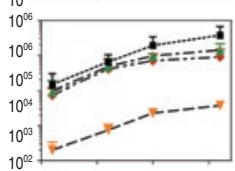

Hep3B

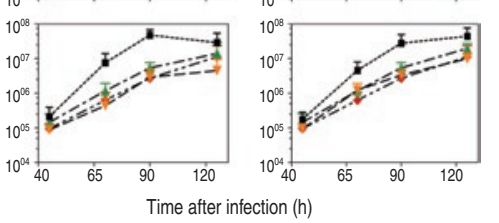

Figure 4 Adp53sensor replication is selectively attenuated in p53-normal cells. (a) Cell lines harboring transcriptionally active p53 (HepG2, HT1080, A549, and MCF-7) or p53-transcriptionally dysfunctional cells (Hep3B, HeLa, Huh7, SAOS, and H1299) were treated with variable doses of doxorubicin as indicated. Eight hours later, cells were adenovirally infected (MOI 0.5). Twenty-four hours after infection, E1A and Gal4-KRAB levels were analyzed by western blot analyses. (b) HepG2 and Huh7 cells were treated with $200 \mathrm{ng} / \mathrm{ml}$ doxorubicin. After 8 hours, cells were infected at an $\mathrm{MOI}$ of 0.005 and incubated for 5 days. Cell layer destruction was investigated by light microscopy. (c) p53-active cells (above) and p53-dysfunctional cells (below) were infected with Adp53sensor, AdCtrl, Onyx-015, or Ad-wt at MOI of 0.005 and treated with or without doxorubicin (200 ng/ml). At different time points, cells were harvested and lysed to determine intracellular viral titers. The results suggest that Adp53sensor replication is selectively attenuated in p53-normal cells. (d) Subcutaneous A549 tumors were intratumorally infected with three sequential injections (indicated by arrows) of $2 \times 10^{8}$ infectious particles of Adp53sensor, AdCtrl, or Ad-LacZ (replication-defective control). Eight hours prior to each tumor infection, mice received a systemic injection of doxorubicin at a final dose of $0.35 \mu \mathrm{g} / \mathrm{g}$ body weight. The tumor growth was determined. The results demonstrate that AdCtrl-infected, p53-positive tumors are more efficiently lysed compared to Adp53sensor. MOI, multiplicity of infection. ${ }^{*} P<0.05$.

p53-positive A549 xenograft model (Figure 4d). Tumor-bearing mice were treated with doxorubicin and intratumorally infected with Adp53sensor, AdCtrl, or Ad-LacZ (a replication-defective virus). In this model, treatment with Adp53sensor seemed to inhibit the growth of A549 tumors more efficiently compared to the replication-incompetent Ad-LacZ (no statistic significance). However, consistent with the p53-selective attenuation of Adp53sensor, we found that Adp53sensor lysed A549 tumors less efficiently compared to AdCtrl. Though the measured difference was statistically significant, the effect was rather moderate. It has to be considered in this model that intratumoral infections usually lead to superinfection of tumor tissue surrounding the point of injection. Superinfection with high multiplicity of infections of conditionally replicating adenoviruses may result in a loss of selectivity. In a subcutaneous model of p53-dysfunctional Huh7 tumors, Adp53sensor and AdCtrl showed a comparable lytic activity (data not shown).

\section{Adp53sensor is highly attenuated in primary hepatocytes in vitro and in vivo}

Tumor cell lines most likely do not reflect the p53-response of normal cells, and active cell cycling can additionally provide a favorable environment for adenoviral replication. Therefore, investigations in these cells may lead to underestimation of the p53-selectivity of Adp53sensor. To address this point in a physiologically more relevant setting, we infected primary human hepatocytes (Figure 5a). Determination of intracellular viral titers revealed a strong inhibition of Adp53sensor replication compared to AdCtrl that was further supported by doxorubicin. Although replication of Onyx-015 was only reduced compared to Ad-wt, Adp53sensor was almost incapable of replication. Interestingly, AdCtrl replication was also significantly reduced compared to the Ad-wt suggesting a constitutionally defective replication of the AdCtrl/Adp53sensor strategy in hepatocytes. This observation might be explained by a delayed activation 

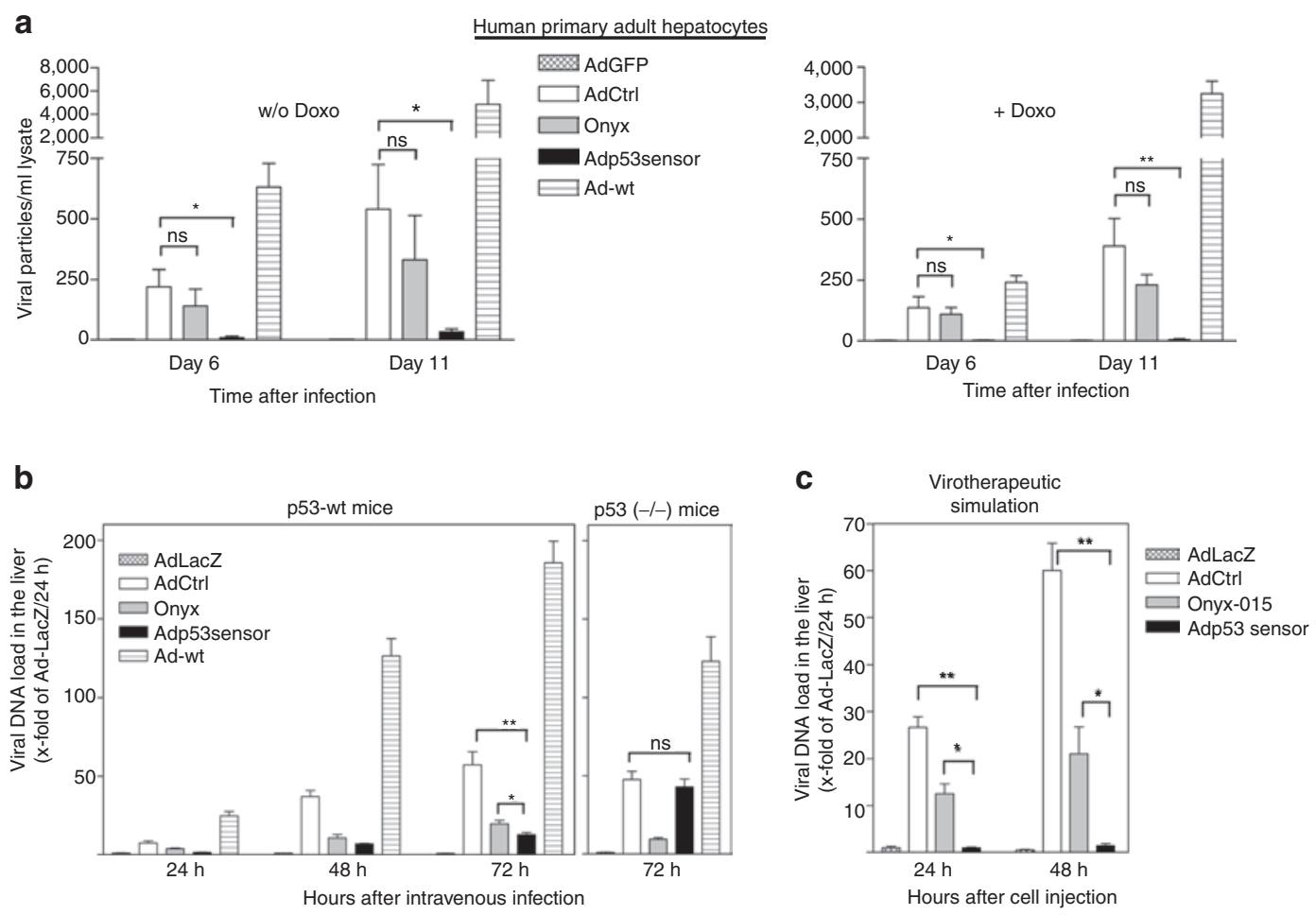

Figure 5 Adp53sensor replication is selectively inhibited in primary human hepatocytes in vitro and in mouse livers in vivo. (a) Primary human hepatocytes were treated with $200 \mathrm{ng} / \mathrm{ml}$ doxorubicin (right panel) or left untreated (left panel). After 8 hours, cells were infected with a replication-defective virus (Ad-GFP), Adp53sensor, AdCtrl, Onyx-015, or Ad-wt at an MOI of 0.005. At the time points indicated, intracellular viral titers were determined. The data show that Adp53sensor replication is almost completely inhibited in primary human hepatocytes. (b) p53selectivity of Adp53sensor replication was investigated in vivo by comparison of viral DNA replication in normal mice and p53-knockout mice after intravenous infection. p53-normal (left) or p53-knockout mice (right) were injected intravenously with $1 \times 10^{7}$ infectious particles. At the indicated time points, DNA was prepared from liver tissue, and the content of viral DNA was determined by qPCR. In normal mice, replication of Adp53sensor was significantly inhibited compared to AdCtrl. Because both viruses replicated comparably in p53-knockout mice, these results confirm in vivo that attenuation of Adp53sensor replication is dependent on p53. (c) For analysis of intrahepatic viral load in a virotherapeutic simulation, Hep3B cells were infected at MOI 25 to achieve almost complete infection. After 24 hours, infected cells were injected into the tail vein of doxorubicin-pretreated mice. At the time points indicated, viral DNA content in the liver was determined as described above. The results show that Adp53sensor DNA replication is almost completely absent in mouse livers in the context of a virotherapeutic simulation. MOI, multiplicity of infection. ${ }^{*} P<0.05,{ }^{* *} P<0.005$.

of the CMV-promoter after viral cell entry in hepatocytes. The CMV-promoter used to control E1A expression in AdCtrl/ Adp53sensor is known to be constitutionally active in tumor cell lines and is also more active compared to the E1A-promoter in transient transfection assays (Figure 1f). In contrast, it has been shown that the CMV-promoter is inactive in resting liver cells and can be reactivated by cellular stress via NFKB-dependent pathways. ${ }^{24}$ Therefore, activation of the CMV-promoter is most likely influenced by the mode of infection that differs between low multiplicity of infection application in vitro and intravenous injection in vivo. It can also not be excluded that the use of the CMV-promoter contributes to the tumor selectivity of Adp53sensor by delayed E1A expression in resting, normal cells.

p53-selective regulation of Adp53sensor was finally addressed in an isogenic setup in vivo. For this purpose, we infected the livers of normal and p53-knockout mice by intravenous administration and determined the intrahepatic load of viral DNA. Though primary murine cells are unable to assemble infectious virions of human adenovirus, the viral genome is normally amplified. To allow proper dissection of virus uptake from replication, the replication-incompetent Ad-LacZ was applied as standard (Figure 5b). Compared to AdCtrl, Adp53sensor DNA load was significantly reduced after infection of p53-normal mice. In contrast, both viruses replicated to the same extent in p53-knockout mice confirming that the control of Adp53sensor replication depends on p53. Though replication of Adp53sensor was not completely inhibited in this experimental setup, selectivity was significantly improved compared to Onyx-015.

The pulsed entry of high particle doses into the liver following intravenous injections results in overinfection of the periphery of liver portal fields that may circumvent the selectivity of conditionally replicating adenoviruses. We therefore investigated the hepatic load of adenoviral DNA in a murine model that provides a steady release of infectious particles into the blood flow that can be expected during virotherapeutic treatments (Figure 5c). Because murine cells do not produce infectious progeny, the consequences of virus release by infected liver cells and subsequent infection of neighboring cells in the human system cannot be taken into account. For our investigations, we injected preinfected Hep3B cells into the tail vein of mice. Hep3B cells were selected because they provide a comparable replication of the investigated 
oncolytic viruses (see Figure 4c). After systemic release of AdCtrl and Onyx-015, we could observe a significantly increasing liver load of adenoviral DNA. In contrast, Adp53sensor DNA levels were almost similar compared to a replication-defective vector

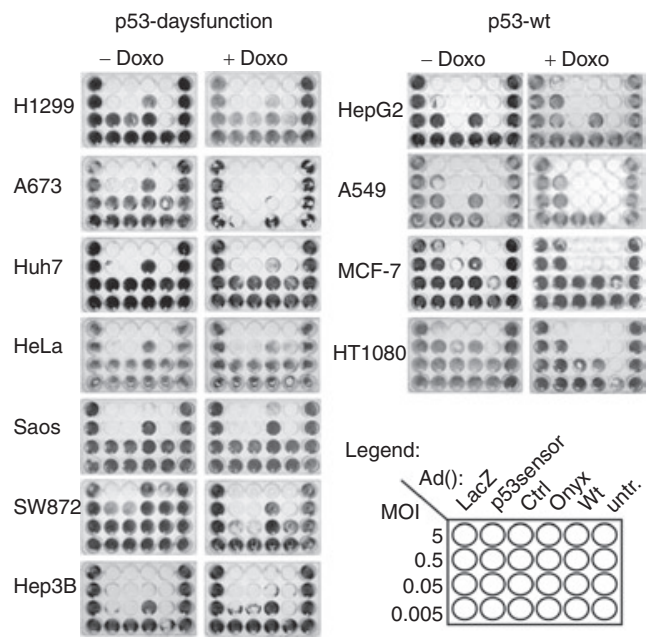

Figure 6 Adp53sensor shows improved p53-selectivity and oncolytic properties in vitro compared to Onyx-015. p53-selectivity of Adp53sensor and lytic potency in p53-inactive tumor cells was analyzed in oncolysis assays. Cells were treated with or without doxorubicin as described in the Materials and Methods section and infected 8 hours later at the indicated MOI. After 5-6 days of incubation, the cell layer destruction was visualized by crystal violet staining. The results demonstrate that Adp53sensor lysed p53-deficient cells more efficiently compared to Onyx-015. Selectivity of Adp53sensor replication and Adp53sensormediated cell lysis was significantly increased after chemotherapeutic triggering. MOI, multiplicity of infection.
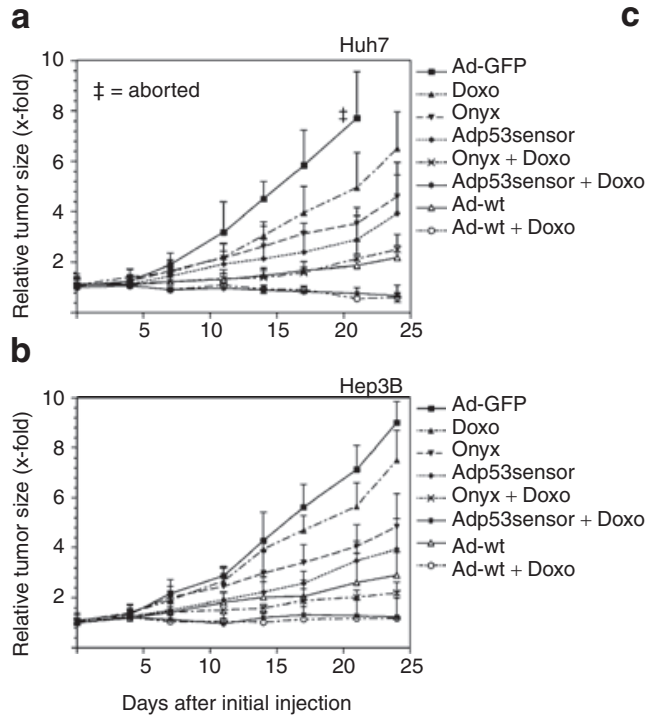

confirming the tight control of Adp53sensor replication under physiologic conditions of a virotherapeutic simulation.

\section{Adp53sensor shows efficient oncolytic properties and demonstrates improved lytic efficacy compared to Onyx-015}

The oncolytic potency as a critical therapeutic parameter was investigated in a panel of tumor cells (Figure 6). In line with the replication data described above, Adp53sensor showed significantly enhanced p53-selectivity compared to Onyx-015, which was further improved by chemotherapeutic triggering. Adp53sensor-mediated oncolysis of p53-dysfunctional cells was generally stronger compared to Onyx-015. The oncolytic potency of Adp53sensor was further confirmed by the observation that the extent of cytolysis was almost as efficient as Ad-wtmediated cytolysis. Irrespective of the p73-status, Adp53sensor showed the same capacity of oncolysis as AdCtrl in all p53dysfunctional cells that were investigated, which is consistent with the inability of p73 to transactivate the p53-dependent promoter prMinRGC after viral infection and chemotherapy as shown above.

\section{Combined Adp53sensor and chemotherapy application inhibits tumor growth in vivo}

To address therapeutic applicability of Adp53sensor in vivo, we investigated oncolytic efficacy in xenotransplant models. As shown in Figure 7a,b, application of Onyx-015 resulted in a growth delay in both xenograft models, whereas doxorubicin hardly reduced the tumor growth. However, doxorubicin significantly improved the efficacy of both virotherapeutic vectors.

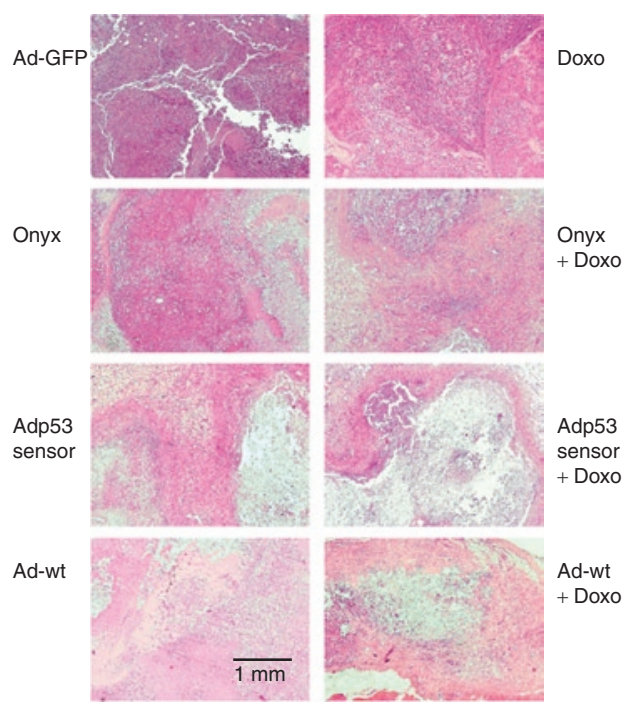

Figure 7 Adp53sensor application and chemotherapeutic treatment lead to effective lysis of human tumor xenografts in vivo. Therapeutic efficacy of virochemotherapy was investigated in vivo in subcutaneously grown tumor cell xenografts on nude mice. Show (a) subcutaneous model of Huh7 cells and (b) Hep3B model. Engrafted tumors were treated once weekly with Ad-wt, Onyx-015, Adp53sensor, or Ad-GFP as replication-defective control, by intratumoral injection alone or in combination with systemic administration of doxorubicin. Tumor growth was assessed for 4 weeks following initial injection. Statistical analysis at day 24 for the Huh7-model: Adp53sensor versus Onyx group ( $P=0.047)$; Adp53sensor versus Adp53sensor/ doxo group $(P=0.0082)$; Adp53sensor versus Onyx/doxo group (ns); wt/doxo versus Adp53sensor/doxo group (ns); wt against Adp53sensor group $(P=0.016)$. Hep3B: Adp53sensor versus Onyx group (ns); Adp53sensor versus Adp53sensor/doxo group $(P=0.005)$; Adp53sensor versus Onyx/doxo group ( $P=0.0172$ ); wt/doxo versus Adp53sensor/doxo group (ns); wt against Adp53sensor group (0.0419). (c) Treated Huh7 tumors were explanted and tumor sections were subjected to histological staining (H\&E) to visualize the extent of tissue destruction. 
a

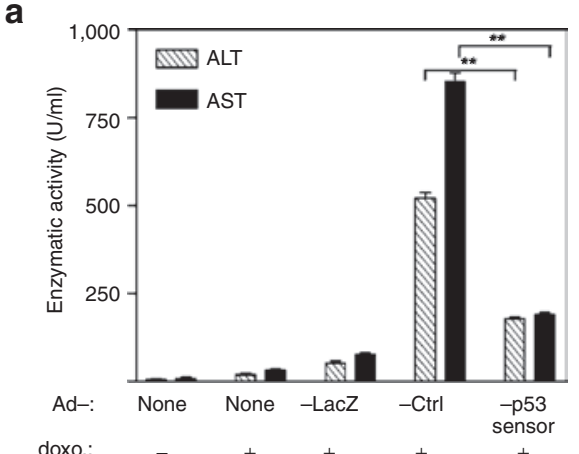

b

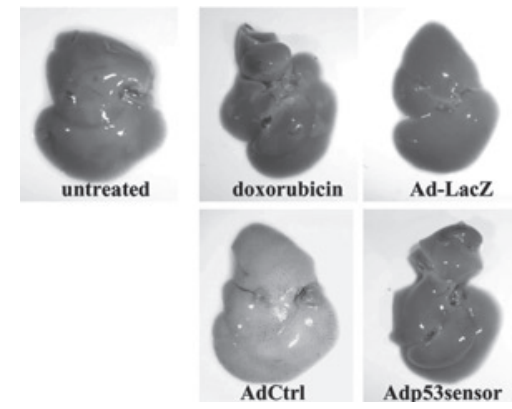

Figure 8 After high-dose intravenous delivery, application of Adp53sensor leads to reduced liver damage in mice. Mice were pretreated with doxorubicin $(1.75 \mu \mathrm{g} / \mathrm{g}$ body weight) 6 hours prior to systemic adenovirus application. Then, $3 \times 10^{8}$ infectious particles of Adp53sensor, AdCtrl, or Ad-LacZ (replication-defective control) were intravenously delivered. (a) Seventy-two hours following infection, mice were harvested, blood samples were drawn, and transaminase activity was determined in the serum. (b) Livers were explanted and macroscopically examined to estimate the extent of liver damage. Our results demonstrate that attenuated replication of Adp53sensor compared to AdCtrl protected the mice from severe liver damage. ${ }^{* *} p<0.005$.

Particularly in Huh7 xenografts, Adp53sensor inhibited tumor growth more efficiently compared to Onyx-015, and was as efficient as the Onyx-015/chemotherapy combination. In the Hep3B-model, the difference between Adp53sensor and Onyx015 was not significant, an observation that could also be due to the HBx expression of Hep3B cells as mentioned above. Finally, the maximum therapeutic effect in both models was achieved after combined treatment with chemotherapy and Adp53sensor, or Ad-wt, respectively. A therapeutic advantage of Ad-wt compared to Adp53sensor could only be observed without chemotherapy. Systemic administration of Adp53sensor or wild type did not result in a significant therapeutic benefit due to relatively poor tumor transduction (data not shown).

Ineffective removal of dead cells and replacement by fibrotic tissue can lead to underestimation of the true therapeutic efficacy when tumor growth curves are studied. Therefore, HE-stained sections were performed from tumor xenografts after defined treatments. Following Onyx-015 treatment, tumors revealed a speckled pattern of necrotic and vital subzones (Figure 7c). Significantly increased tissue destruction and larger fields of advanced necrosis could be observed after treatment with Adp53sensor alone or after Onyx-015/chemotherapy combination. In contrast, tumor nodules that remained after chemotherapy/Adp53sensor virotherapy predominantly consisted of scar tissue containing loosely dispersed cells of fibrotic morphology.

\section{p53-Selective attenuation of Adp53sensor results in reduced liver damage}

During virotherapy, a significant proportion of the released adenoviral progeny can be flushed out of the tumor bed thereby threatening the liver as the predominant target organ of systemic adenoviral infections. Tight replication control of oncolytic adenoviruses is therefore an important prerequisite for the protection of the liver. To address the question of hepatic toxicity directly, doxorubicin-pretreated mice were intravenously infected. After 3 days, blood samples were drawn and serum transaminase activities were determined (Figure 8a). Explanted livers were investigated for macroscopic signs of liver damage (Figure $\mathbf{8 b}$ ). Livers from mice that received only doxorubicin or Ad-LacZ/doxorubicin were undistinguishable from untreated livers. Though ALT/AST levels were moderately elevated after infection with Adp53sensor compared to doxorubicin alone or Ad-LacZ, no significant liver damage was macroscopically detectable. In contrast, a severe liver damage and a dramatic increase of serum transaminase activity could be observed after infection by AdCtrl. Together, our results confirm antitumoral efficacy of Adp53sensor and indicate an improved protection against systemic side effects during Adp53sensor virotherapy.

\section{DISCUSSION}

Inhibition of p53-transcriptional activity represents a hallmark of almost all cancers. Consequently, a strategy that restricts viral replication to cells with p53-transcriptional dysfunction would provide means for constructing oncolytic vectors for the majority of human cancers. Some concepts have already been developed for targeting of p53-alterations by viruses. Most prominent in this regard are Onyx-015 and existing variants. However, p53specificity of Onyx-015 has been questioned by several groups, ${ }^{25-28}$ and other studies suggested that E1B-55k-mediated degradation of p53 is not necessary to allow for adenoviral replication. ${ }^{29,30}$ In another study, the adenovirus 01/PEME has been presented that targets both p53-dysfunctional and E2F-active tumors. ${ }^{31}$ Though 01/PEME-replication was attenuated in primary human fibroblasts, replication was not inhibited in A549 and HepG2 cells, despite transcriptionally active p53. In contrast, we demonstrate that replication of Adp53sensor was significantly inhibited in these cell lines thus confirming the stringency of p53-dependent E1A repression. In 01/PEME, p53-dependent inhibition of viral replication was only achieved when an $\mathrm{N}$-terminally variant of E1A was used that reduces negative interference with p53-dependent transactivation. Our data suggest that the E1A silencing by targeted repression used for Adp53sensor is sufficient to avoid negative interference of viral proteins with $\mathrm{p} 53$-transcriptional activity and thus allowed application of wild-type viral genes for an unaltered course of the viral life cycle. In contrast to Onyx-015 and 01/PEME, we could show that the p53-specificity of Adp53sensor solely relies on the presence of p53-dependent transcription in target cells. Addressing the safety of Adp53sensor application, we further demonstrated that Adp53sensor led to reduced viral load in mice livers during virotherapeutic simulations and that mice were protected from 
severe liver damage following administration of high systemic adenovirus doses. A central question for the applicability of oncolytic viruses targeting p53-transcriptional deficiency is the role of other p53 family members that can potentially compensate for the lack of p53-transcriptional functions. In contrast to p53, the p53-homologues, p73 and p63, are rarely mutated in tumors. ${ }^{32}$ However, as recently confirmed by studies carried out in TAp73 knockout mice, only the transactivating isoforms can be classified as tumor suppressors. ${ }^{33}$ Although p53 primarily acts as a tumor suppressor in stress response pathways, mice with complete p73or p63-knockout have shown that these proteins preferably play an important role in differentiation and embryonal development than in protection against cancer. ${ }^{34-36}$ On the other hand, studies have revealed that mutant $\mathrm{p} 53$ or $\Delta \mathrm{Np} 63$ can interact and downregulate the function of $\mathrm{p} 73$, providing a molecular explanation for the gain of function of p53-mutations and suggesting a role of p73 and p63 in carcinogenesis. ${ }^{37-40}$ Several groups have reported that p73 and p63 are induced by cisplatin or doxorubicin. ${ }^{15,16,19,38,39,41}$ Additionally, inactivation of p73 and p63 confers p53-independent chemoresistance, at least in part due to the lack of p73-and p63-mediated transactivation of proapoptotic genes like AIP1, BAX, CD95, and PUMA. ${ }^{14}$ The prMinRGC-promoter used in Adp53sensor showed a comparable susceptibility for transactivation by $\mathrm{p} 73 \beta$ or $\mathrm{p} 53$, when these proteins were overexpressed. Regarding the endogenous situation, nuclear $\mathrm{p} 73 \beta$-levels following adenoviral infection or chemotherapy appeared to be insufficient to trigger a transcriptional response like p53. A p73-knockdown in p53-deficient cells rather suggested an inhibitory function of endogenous p73 on the p53-responsive promoter used in this study. Consistent with the inability of endogenous p73 to activate the prMinRGC-promoter after stimulation, the oncolytic efficacy of Adp53sensor was not compromised in any of the investigated p53-dysfunctional cells, regardless of the cellular p73-status. Our results therefore indicate that p53-transcriptional dysfunction is a well-suited, broad-range target for therapy of HCC and is not restricted by other p 53 family members. Finally, a general advantage of the Adp53sensor strategy is the applicability for the design of further oncolytic viruses because it should be transferable to other DNA viruses such as CMV or HSV. Thus, the strategy implemented in Adp53sensor could provide a general method for engineering a broad spectrum of p53-dependent oncolytic viruses for the treatment of p53-dysfunctional tumors.

\section{MATERIALS AND METHODS}

Cells. Human Hep3B, HepG2, HT1080, A549, H1299, SAOS-2, MCF-7, HeLa, and 293 cells were obtained from ATCC (Rockville, MD). Huh7 cells were obtained from the JCRB (Osaka, Japan). A673 and SW872 were provided by the CNIO (Madrid, Spain). Human primary adult hepatocytes were purchased from Cytonet (Hannover, Germany).

Adenoviruses and plasmids. The plasmids pcDNA3-HA-p73a and pcDNA3-HA-p73 $\beta$ were kindly provided by W.G. Kaelin. The plasmids pC53SN3 and pSK-45-13-2-PyCAT were kind gifts by B. Vogelstein. The plasmid pZIK $\triangle \mathrm{BH}$ was kindly provided by L. Lania. pAdApt was kindly given by R. Hoeben.

The vectors prMinRGC-GAL4KRAB, prMinRGC-Luc, prMinConLuc, and $\mathrm{CMV}_{\text {gal }}$-Luc have been described previously. ${ }^{12}$

For construction of the plasmid prE1A-Luc, the Ad5-E1A-promoter (pos. 2-558) was PCR-amplified from adenoviral DNA (primers: 5'-CC
GAGCTCATCAATAATATACCTTATTTTGGATTGAAGCC-3' and 5'GTAAGCTTCAGTCCCGGTGTCGGAGCGG-3') and cloned into pGL2Basic (Promega, Mannheim, Germany).

The bicistronic adenoviral vectors Adp53sensor and AdCtrl were constructed on the basis of the shuttle plasmid pHM3 containing a 3'$\mathrm{CMV}_{\text {gal }} / \mathrm{E} 1 / \mathrm{IRES} / \mathrm{EGFP} / \mathrm{pA}$ followed by a $5^{\prime}$-prMinRGC-GAL4KRAB/pA expression unit (cloning details upon request). For the generation of the p53-independent replicating AdCtrl, a promoterless Gal4-KRAB-cassette was used. pHM3-CMV gal $_{\text {E1/IRES/EGFP/pA/(prMinRGC)-GAL4KRAB/ }}$ $\mathrm{pA}$ constructs were released from the plasmid backbone by digesting $\mathrm{PI}-\mathrm{Sce}$ and I-CeuI, and recombinant adenoviruses were constructed according to a ligation method described previously. ${ }^{42}$ This construction finally yielded adenoviruses of an approximate length of 36.8 kilobases (kb) (Adp53sensor) and $36.3 \mathrm{~kb}$ (AdCtrl). Viruses were propagated in 293 cells according to standard procedures. In general, significantly lower yields were obtained compared to standard adenoviruses such as Ad-wt. Because preparations were checked by PCR for eventual loss of heterologous DNA fragments and for the length of repetitive sequences, we could not find significant evidence for genetic instability or formation of shortened, deregulated viruses.

Luciferase assays. $6 \times 10^{5}$ cells were seeded in $60 \mathrm{~mm}$ dishes the day before transfection. Cells were transfected using the calcium phosphate precipitation method or Lipofectamine (Invitrogen, Carlsbad, CA). Generally, $1 \mu \mathrm{g}$ luciferase reporter plasmid (prMinRGC-Luc, prMinCon-Luc, prE1A-Luc) and $0.3 \mu \mathrm{g}$ pCMV-LacZ for normalization were adjusted with pBluescript KS (Stratagene, La Jolla, CA) to $5 \mu$ g DNA per dish. Chemotherapeutic/ adenovirus treatment was performed as described in the figure legends. Forty-eight hours after transfection, extracts were obtained according to standard protocols, luciferase activity was determined, and luciferase measurements were normalized by $\beta$-galactosidase assay.

RNA interference. siRNA-mediated knockdown of p73 was evaluated by real-time quantitative PCR. $5 \times 10^{5} \mathrm{H} 1299$ cells were transfected twice with 40 pmol siRNA using HiPerfect (Qiagen, Hilden, Germany). Twentyfour hours after transfection, doxorubicin was added $(200 \mathrm{ng} / \mathrm{ml})$. After 48 hours, total RNA was extracted using peqGOLD-RNAPure (PeqLab, Erlangen, Germany). Reverse transcription was performed using the TaqMan-Kit (random hexamer as primer; Applied Biosystems, Foster City, CA) followed by DNase-digestion. cDNA was subjected to quantitative PCR using SYBR Green in an ABI 7300 cycler (Applied Biosystems). Quantitative PCR for determination of total p73 was performed using primers against the DBD (5'-GTGACCGACGTCGTGAAACGCTGC-3' and $5^{\prime}$-CTGGCTGGAGCAGACTGTCCTTCG-3'). Transactivating p73 isoforms were quantified using primers against the transcriptional activation domain (5'-CAGACAGCACCTACTTCGACCTTCC-3' and 5'-GGA AGACGTCCATGCTGGAATCCG-3'). Ct-values were normalized against GAPDH. Used siRNAs against all p73 isoforms were directed against the sequences 5'-CTCGGGAGGGACTTCAACGAA-3" ("DBD"). siRNA against transactivating p73 isoforms was described previously. ${ }^{43}$ The p53siRNA, MDM2-siRNA, and the "negative control siRNA" were purchased from Qiagen. For siRNA-mediated knockdown, co-transfection assays with prMinRGC-Luc as reporter and CMV-LacZ as internal control were performed using Lipofectamine 2000 (Invitrogen).

Western blot analysis. Whole cell extracts were prepared using radioimmunoprecipitation assay buffer according to standard protocols. For nuclear extract preparation, the cell pellet was resuspended in $200 \mu \mathrm{lNB}$ ( $10 \mathrm{mmol} / \mathrm{l}$ TrisCl pH 7.4, $2 \mathrm{mmol} / 1 \mathrm{MgCl}_{2}, 140 \mathrm{mmol} / \mathrm{l} \mathrm{NaCl}$ ) supplemented with $0.1 \%$ Triton-X100. The nuclei were centrifuged through a $50 \%$ sucrose cushion in NPB $\left(10\right.$ minutes, $\left.4^{\circ} \mathrm{C}, 15,000 \mathrm{~g}\right)$. The supernatant was removed and nuclei were lysed with radioimmunoprecipitation assay. Ten micrograms of protein (for detection of p73: $100 \mu \mathrm{g}$ ) were separated on a 10\% SDS gel and blotted onto a HyBond membrane (Millipore, Billerica, MA). Loading was controlled by Ponceau-Red staining (nuclear fractions) 
and $\beta$-actin-blot (whole cell extracts). Used primary antibodies were as follows: anti-p53 Ab-6 (Calbiochem, Nottingham, UK); anti-p73 antibody (BL906; Bethyl Laboratories, Montgomery, TX); and anti-Gal4-DBD and anti-Ad2 E1A (Santa Cruz, Santa Cruz, CA). Bands were visualized using ECL (Amersham, Freiburg, Germany).

Quantitative analysis of viral titers. For measurement of viral replication kinetics, $0.5-1 \times 10^{6}$ cells were plated and grown to subconfluency. On the day of infection, the medium was exchanged by medium containing $2 \%$ fetal bovine serum \pm doxorubicin. After 8 hours, cells were adenovirally infected. At the time points indicated in the figures, cells were harvested in $0.5 \mathrm{ml}$ phosphate-buffered saline, viral particles were released by repeated freezing/thawing and titered by rapid titer assay (BD Biosciences, San Jose, CA).

Oncolysis assays. Depending on cell size and proliferation speed, cells were seeded in 24-well plates at a density of $0.5 \times 10^{4}$ to $2.5 \times 10^{5}$ per well and grown for subconfluency at the time of infection. Doxorubicin was then applied at concentrations that allow cell survival for $\sim 1$ week: $200 \mathrm{ng} / \mathrm{ml}$ (HepG2, A549); 150 ng/ml (MCF-7, Huh7); 100 ng/ml (U2-OS, H1299, SW872, HeLa, Hep3B, HT1080); and $50 \mathrm{ng} / \mathrm{ml}$ (SAOS-2, A673). After 8 hours, adenovirus was added. After 5-6 days, cell layer destruction was visualized by crystal violet staining.

Animal experiments. Six-week-old, homozygous p53-knockout mice (inbred from strain B6.129S2-Trp53 ${ }^{\text {tmlTyi }} /$ J; Jackson Laboratory, Bar Harbor, ME) were provided by the Central Animal Facility at the Helmholtz Centre of Infection Research (HZI, Braunschweig, Germany). Six-week-old C57BL/6 and NMRI nu/nu mice were obtained from the Animal Research Institute of the Hannover Medical School. All animal experiments were performed according to the German legal requirements. To examine the therapeutic efficacy, tumor xenografts were established by subcutaneous injection of $1 \times 10^{7}$ cells (Hep3B, H1299) into both flanks of mice. Tumor nodules were grown to a size of $\sim 250 \mathrm{~mm}^{3}$. As chemotherapy, mice received i.v. injections of $1.75 \mu \mathrm{g}$ doxorubicin/g body weight once a week. Intratumoral treatments with adenovirus were performed on days 0,7 , 14 , and 21 by injection of $1 \times 10^{9}$ infection forming units. For statistical tumor growth assessment, infected tumors were measured twice a week. Tumor volume was calculated using the equation: $\mathrm{V}$ (tumor) = (length $\times$ width $\left.^{2}\right) / 2$. For each group, six tumors were assessed. At the end of the experiment, paraffin sections of the tumors were stained by hematoxylin/ eosin. Microscopic images were made at 40 -fold magnification with a Zeiss Axiom-mcR camera (Carl Zeiss, Jena, Germany) and processed using AxioVision software (Carl Zeiss).

To determine Adp53sensor selectivity in a tumor model in vivo, nude mice were subcutaneously inoculated with $1 \times 10^{7}$ A549 cells, and tumors were grown to a size of $\sim 200 \mathrm{~mm}^{3}$. Tumors were infected and treated with doxorubicin as described in the figure legend.

Determination of intrahepatic viral load and liver damage. For analysis of intrahepatic viral DNA following systemic adenoviral infection, mice were pretreated intravenously with doxorubicin $(1.75 \mu \mathrm{g} / \mathrm{g}$ bodyweight), 6 hours prior to adenovirus injection. For the analysis of intrahepatic viral DNA during a virotherapeutic simulation, Hep3B cells were infected at multiplicity of infection of 25 (almost complete infection). After 24 hours, $5 \times 10^{5}$ cells were injected i.v. in doxorubicin-pretreated mice (performed as described above). At the time points indicated in the figures, mice were killed, and DNA from liver tissue was purified using the QIAmp-DNA-Mini Kit (Qiagen). Viral DNA quantification was performed by real-time PCR (qPCR MasterMix Plus; Eurogentec, Seraing, Belgium) using $100 \mathrm{ng}$ of liver DNA and adenoviral hexonspecific primer probes as described before. ${ }^{44} 18 \mathrm{~S}$ Genomic Control Kit (Eurogentec) served as internal control. For the investigation of liver damage, explanted livers were examined macroscopically, and ALT/AST activities were determined in serum samples from treated mice using the AST/ALT enzymatic assay kit (Bioo Scientific) according to the manufacturer's protocols.

Statistics. Two groups were compared by unpaired $t$-test. $P<0.05$ was considered as statistically significant.

\section{ACKNOWLEDGMENTS}

Research was supported by grants of the EU Commission (contract no. 12948 "Netsensor"), the German Research Council, DFG (KFO119/1-2), TRR77, and the Mildred-Scheel-Stiftung.

\section{REFERENCES}

1. Hurtado Picó, A, Wang, X, Sipo, I, Siemetzki, U, Eberle, J, Poller, W et al. (2005). Viral and nonviral factors causing nonspecific replication of tumor- and tissue-specific promoter-dependent oncolytic adenoviruses. Mol Ther 11: 563-577.

2. Bilsland, AE, Merron, A, Vassaux, G and Keith, WN (2007). Modulation of telomerase promoter tumor selectivity in the context of oncolytic adenoviruses. Cancer Res 67: 1299-1307.

3. Levine, AJ (1997). p53, the cellular gatekeeper for growth and division. Cell 88 : 323-331.

4. Fridman, JS and Lowe, SW (2003). Control of apoptosis by p53. Oncogene 22: 9030-9040.

5. Harada, JN and Berk, AJ (1999). p53-Independent and -dependent requirements for E1B-55K in adenovirus type 5 replication. / Virol 73: 5333-5344.

6. O'Shea, CC, Soria, C, Bagus, B and McCormick, F (2005). Heat shock phenocopies E1B-55K late functions and selectively sensitizes refractory tumor cells to ONYX-015 oncolytic viral therapy. Cancer Cell 8: 61-74.

7. Zheng, X, Rao, XM, Gomez-Gutierrez, JG, Hao, H, McMasters, KM and Zhou, HS (2008). Adenovirus E1B55K region is required to enhance cyclin E expression for efficient viral DNA replication. / Virol 82: 3415-3427.

8. Thomas, MA, Broughton, RS, Goodrum, FD and Ornelles, DA (2009). E4orf1 limits the oncolytic potential of the E1B-55K deletion mutant adenovirus. / Viro/ 83: 2406-2416.

9. Lowe, SW, Bodis, S, McClatchey, A, Remington, L, Ruley, HE, Fisher, DE et al. (1994). p53 status and the efficacy of cancer therapy in vivo. Science 266: 807-810.

10. Kubicka, S, Kühnel, F, Zender, L, Rudolph, KL, Plümpe, J, Manns, M et al. (1999). p53 represses CAAT enhancer-binding protein (C/EBP)-dependent transcription of the albumin gene. A molecular mechanism involved in viral liver infection with implications for hepatocarcinogenesis. J Biol Chem 274: 32137-32144.

11. Wirth, T, Kühnel, F, Fleischmann-Mundt, B, Woller, N, Djojosubroto, M, Rudolph, KL et al. (2005). Telomerase-dependent virotherapy overcomes resistance of hepatocellular carcinomas against chemotherapy and tumor necrosis factor-related apoptosis-inducing ligand by elimination of Mcl-1. Cancer Res 65: 7393-7402.

12. Kühnel, F, Zender, L, Wirth, T, Schulte, B, Trautwein, C, Manns, M et al. (2004). Tumor-specific adenoviral gene therapy: transcriptional repression of gene expression by utilizing p53-signal transduction pathways. Cancer Gene Ther 11: 28-40.

13. Shimada, A, Kato, S, Enjo, K, Osada, M, Ikawa, Y, Kohno, K et al. (1999). The transcriptional activities of p53 and its homologue p51/p63: similarities and differences. Cancer Res 59: 2781-2786.

14. Flores, ER, Tsai, KY, Crowley, D, Sengupta, S, Yang, A, McKeon, F et al. (2002). p63 and $\mathrm{p} 73$ are required for $\mathrm{p} 53$-dependent apoptosis in response to DNA damage. Nature 416: 560-564.

15. Agami, R, Blandino, G, Oren, $M$ and Shaul, Y (1999). Interaction of c-Abl and p73alpha and their collaboration to induce apoptosis. Nature 399: 809-813.

16. Gong, JG, Costanzo, A, Yang, HQ, Melino, G, Kaelin, WG Jr., Levrero, M et al. (1999). The tyrosine kinase c-Abl regulates p73 in apoptotic response to cisplatin-induced DNA damage. Nature 399: 806-809.

17. Yuan, ZM, Shioya, H, Ishiko, T, Sun, X, Gu, J, Huang, YY et al. (1999). p73 is regulated by tyrosine kinase $\mathrm{c}-\mathrm{Abl}$ in the apoptotic response to DNA damage. Nature 399: 814-817.

18. Steegenga, WT, Shvarts, A, Riteco, N, Bos, JL and Jochemsen, AG (1999). Distinct regulation of $\mathrm{p} 53$ and $\mathrm{p} 73$ activity by adenovirus E1A, E1B, and E4orf6 proteins. Mol Cell Biol 19: 3885-3894.

19. Gressner, O, Schilling, T, Lorenz, K, Schulze Schleithoff, E, Koch, A Schulze-Bergkamen, $\mathrm{H}$ et al. (2005). TAp63alpha induces apoptosis by activating signaling via death receptors and mitochondria. EMBO / 24: 2458-2471.

20. Lin, KW, Nam, SY, Toh, WH, Dulloo, I and Sabapathy, K (2004). Multiple stress signals induce p73beta accumulation. Neoplasia 6: 546-557.

21. Sayan, AE, Paradisi, A, Vojtesek, B, Knight, RA, Melino, G and Candi, E (2005). New antibodies recognizing p73: comparison with commercial antibodies. Biochem Biophys Res Commun 330: 186-193.

22. Lokshin, M, Tanaka, T and Prives, C (2005). Transcriptional regulation by $\mathrm{p} 53$ and p73. Cold Spring Harb Symp Quant Biol 70: 121-128.

23. Schaack, J, Maguire, HF and Siddiqui, A (1996). Hepatitis B virus X protein partially substitutes for E1A transcriptional function during adenovirus infection. Virology 216 : 425-430.

24. Löser, P, Jennings, GS, Strauss, M and Sandig, V (1998). Reactivation of the previously silenced cytomegalovirus major immediate-early promoter in the mouse liver: involvement of NFkappaB. J Virol 72: 180-190.

25. Hall, AR, Dix, BR, O'Carroll, SJ and Braithwaite, AW (1998). p53-dependent cell death/apoptosis is required for a productive adenovirus infection. Nat Med $\mathbf{4}$ 1068-1072.

26. Rothmann, T, Hengstermann, A, Whitaker, NJ, Scheffner, M and zur Hausen, H (1998) Replication of ONYX-015, a potential anticancer adenovirus, is independent of p53 status in tumor cells. J Virol 72: 9470-9478. 
27. Dix, BR, O'Carroll, SJ, Myers, Cl, Edwards, SI and Braithwaite, AW (2000). Efficient induction of cell death by adenoviruses requires binding of E1B55k and p53. Cancer Res 60: 2666-2672

28. O'Shea, CC, Johnson, L, Bagus, B, Choi, S, Nicholas, C, Shen, A et al. (2004). Late vira RNA export, rather than p53 inactivation, determines ONYX-015 tumor selectivity. Cancer Cell 6: 611-623.

29. Hobom, U and Dobbelstein, M (2004). E1B-55-kilodalton protein is not required to block p53-induced transcription during adenovirus infection. J Virol $\mathbf{7 8}$ : 7685-7697.

30. Koch, P, Gatfield, I, Löber, C, Hobom, U, Lenz-Stöppler, C, Roth, I et al. (2001). Efficient replication of adenovirus despite the overexpression of active and nondegradable p53. Cancer Res 61: 5941-5947.

31. Ramachandra, M, Rahman, A, Zou, A, Vaillancourt, M, Howe, JA, Antelman, D et al. (2001). Re-engineering adenovirus regulatory pathways to enhance oncolytic specificity and efficacy. Nat Biotechnol 19: 1035-1041.

32. Kaelin, WG Jr. (1999). The p53 gene family. Oncogene 18: 7701-7705.

33. Tomasini, R, Tsuchihara, K, Wilhelm, M, Fujitani, M, Rufini, A, Cheung, CC et al. (2008). TAp73 knockout shows genomic instability with infertility and tumor suppressor functions. Genes Dev 22: 2677-2691.

34. Mills, AA, Zheng, B, Wang, XJ, Vogel, H, Roop, DR and Bradley, A (1999). p63 is a p53 homologue required for limb and epidermal morphogenesis. Nature 398: 708-713.

35. Yang, A, Schweitzer, R, Sun, D, Kaghad, M, Walker, N, Bronson, RT et al. (1999). p63 is essential for regenerative proliferation in limb, craniofacial and epithelial development. Nature 398: 714-718.
36. Yang, A, Walker, N, Bronson, R, Kaghad, M, Oosterwegel, M, Bonnin, I et al. (2000). p73-deficient mice have neurological, pheromonal and inflammatory defects but lack spontaneous tumours. Nature 404: 99-103.

37. Gaiddon, C, Lokshin, M, Ahn, J, Zhang, T and Prives, C (2001). A subset of tumor-derived mutant forms of p53 down-regulate p63 and p73 through a direct interaction with the $\mathrm{p} 53$ core domain. Mol Cell Biol 21: 1874-1887.

38. Bergamaschi, D, Gasco, M, Hiller, L, Sullivan, A, Syed, N, Trigiante, G et al. (2003). p53 polymorphism influences response in cancer chemotherapy via modulation of p73-dependent apoptosis. Cancer Cell 3: 387-402.

39. Irwin, MS, Kondo, K, Marin, MC, Cheng, LS, Hahn, WC and Kaelin, WG Jr. (2003). Chemosensitivity linked to p73 function. Cancer Cell 3: 403-410.

40. Rocco, JW, Leong, CO, Kuperwasser, N, DeYoung, MP and Ellisen, LW (2006). p63 mediates survival in squamous cell carcinoma by suppression of p73-dependent apoptosis. Cancer Cell 9: 45-56.

41. Costanzo, A, Merlo, P, Pediconi, N, Fulco, M, Sartorelli, V, Cole, PA et al. (2002). DNA damage-dependent acetylation of p73 dictates the selective activation of apoptotic target genes. Mol Cell 9: 175-186.

42. Mizuguchi, $\mathrm{H}$ and Kay, MA (1998). Efficient construction of a recombinant adenovirus vector by an improved in vitro ligation method. Hum Gene Ther 9: 2577-2583.

43. Vayssade, M, Haddada, H, Faridoni-Laurens, L, Tourpin, S, Valent, A, Bénard, J et al. (2005). P73 functionally replaces p53 in Adriamycin-treated, p53-deficient breast cancer cells. Int / Cancer 116: 860-869.

44. Zhang, YA, Nemunaitis, J, Samuel, SK, Chen, P, Shen, Y and Tong, AW (2006) Antitumor activity of an oncolytic adenovirus-delivered oncogene small interfering RNA. Cancer Res 66: 9736-9743. 